Rev. SINAPSIS, Vol. 7, N 1, Diciembre 2015

ISSN 1390 - 9770

\title{
Sistema Informático para la gestión de servicios médicos de la Fundación Padre Miguel Fitzgerald del Cantón Chone.
}

\section{Sistema Informático para la gestión de servicios médicos.}

Daniela Vera Vélez, Mg. IE. $\left.{ }^{1}\right)$

Cristian Mera Macías, Mg. IE. $\left(^{2}\right)$

( $\left.{ }^{1}\right)$ Universidad Laica Eloy Alfaro de Manabí Extensión Chone, Chone, Ecuador.

( $\left.{ }^{2}\right)$ Universidad Laica Eloy Alfaro de Manabí Extensión Chone, Chone, Ecuador.

Contacto: angel.lmera@uleam.edu.ec

Receptado: 02/09/2015 Aceptado: 09/12/2015

\section{Resumen}

El objetivo de esta investigación fue analizar la realidad de la Fundación Padre Miguel Fitzgerald del Cantón Chone en cuanto a los servicios médicos que ofrece, a partir de una encuesta aplicada a 343 clientes externos o pacientes y 15 clientes internos o empleados de la entidad, una vez analizada dicha problemática se procedió a construir un sistema informático con la finalidad de mejorar la gestión de servicios médicos, para esto se recopiló la información necesaria para construir una aplicación informática hecha a la medida del problema encontrado, con la aplicación construida se procedió a implementarla para proceder con la evaluación respectiva luego de un tiempo prudencial, una vez que se contó con la información suficiente registrada en el sistema se evaluó el nivel de satisfacción de los usuarios, dando como resultado niveles "muy satisfactorios" de aceptación para el sistema informático implementado.

Sistema Informático para la gestión de servicios médicos. 
Rev. SINAPSIS, Vol. 7, Nº 1, Diciembre 2015

ISSN 1390 - 9770

Palabras clave: Aplicación informática, sistema computacional, gestión de salud, servicios médicos, software de salud.

Computer system for the management of medical services of the Father Miguel Fitzgerald Foundation of Chone Canton.

\begin{abstract}
The objective of this research was to analyze the reality of Father Miguel Fitzgerald Foundation of Chone Canton regarding the medical services it offers, from a survey of 343 patients and 15 employees of the entity, once this problem analyzed it proceeded to build a computer system in order to improve the management of medical services, for this necessary information is collected to build a software application tailored to the problem encountered with the application built it proceeded to implement to proceed with the respective evaluation after a reasonable time, once they had sufficient information recorded in the system the level of user satisfaction was evaluated, resulting in "very satisfactory" levels of acceptance for the implemented computer system.
\end{abstract}

Keyword: Computer application, computer system, health management, medical services, health software

\title{
Introducción
}

Según (Stusser \& Rodríguez, 2006) las primeras aplicaciones de la informática en el sector de la salud fueron los sistemas de recolección continua, la comunicación y la recuperación computarizada de los datos médicos de los pacientes y familiares en la APS (atención primaria de salud). Esto llevó a estudiar la clasificación de enfermedades que había surgido en los hospitales para ajustarla a las necesidades del trabajo médico más ambulatorio en la APS. También, hizo trabajar con un universo de trabajo por área de residencia, en vez de la casuística hospitalaria generalmente por enfermedades. Los sistemas continuos sugirieron hacer investigaciones esporádicas.

Sistema Informático para la gestión de servicios médicos. 
A nivel mundial un gran número de empresas han optado por automatizar los procesos que controlan, en el sector de la salud la realidad no es diferente, es así que revisando la literatura existente se han encontrado varias investigaciones referentes al desarrollo de sistemas informáticos para la gestión de los servicios médicos. (Cabañas, Scholer, \& Tintinalli, 2008) afirman que la gestión, procesamiento y aplicación de los datos de la atención prestada a los pacientes así como los datos operativos están transformando y mejorando los sistemas de atención pre hospitalarias y las intervenciones de los servicios de urgencias hospitalarios, siendo justamente por este motivo que la utilización de la tecnología juega un papel muy importante ya que nos permiten realizar las actividades de manera ágil y precisa, ofreciendo la posibilidad de aprovechar la información generada para soportar la toma de decisiones en una determinada entidad.

Lo anteriormente expuesto se corrobora con lo expresado por (Avella \& Parra, 2013) quienes afirman que el uso de las Tecnologías de la Información y la Comunicación (TIC), en la atención en salud constituye una herramienta para mejorar el impacto de las intervenciones en la comunidad, permitiendo un acceso más equitativo y eficiente a los servicios, mejorando la oportunidad de la atención y la costoefectividad de los tratamientos.

Asimismo (Llanusa, Rojo, Caraballoso, Capote, \& Perez, 2005) manifiestan que con el uso de las TICs (Tecnologías de la Información y de la comunicación) se ofrece una oportunidad sin precedentes, que sirve de base para la auto preparación de especialistas, ya que la atención primaria de la salud constituye en la actualidad un pilar fundamental en la estrategia de salud, concluyendo que por medio de este eje esencial se prevé elevar la resolutividad, cobertura y accesibilidad al sistema de salud.

Las redes de asistencia pública en los actuales momentos están divididas en hospitales y centros de salud. El principal problema que tienen las redes de salud es la escasez de personal y su limitada capacidad de resolución en atención primaria y especializada ya que por la demanda diaria de pacientes el tiempo de labor se vuelve muy escaso acotando a esto la falta de espacios y medicinas en muchos hospitales del país. Por otra parte está el problema de atención al usuario, que muchas veces por la falta de procesos claros y la escasa utilización de las TICs para apoyar sus actividades, se dan muchos 
problemas relacionados con la demora, la aglomeración de documentos y las limitaciones para emitir información importante que soporte de manera apropiada la toma de decisiones.

Entre los servicios médicos más esenciales, están las historias clínicas o HC, (Ribelles, y otros, 2010) manifiestan que la Historia Clínica de un paciente se define como el conjunto de documentos que contienen los datos, valoraciones e informaciones de cualquier índole sobre la situación y la evolución clínica de un paciente a lo largo del proceso asistencial. Actualmente, el soporte habitual de recogida de esta información continúa siendo el papel, si bien la natural integración de la tecnología con la documentación médica ha originado avances significativos en los sistemas de registros médicos desde que en la década de los sesenta Weed estableciera el concepto de "Historia Clínica electrónica".

Los problemas inherentes a la gestión de historial clínicas en papel se encuentran en la Fundación Padre Miguel Fitzgerald del Cantón Chone, por esta razón surge la siguiente interrogante: ¿Cómo mejorar la gestión de servicios médicos de la Fundación Padre Miguel Fitzgerald del Cantón Chone?, por tanto este trabajo ha buscado implementar una mejora desde el punto de vista tecnológico, específicamente mediante un sistema informático caracterizado por poseer módulos como datos primarios, historias clínicas, registro de exámenes, control de servicios y control de citas para mejorar la gestión de servicios médicos de la Fundación Padre Miguel Fitzgerald del Cantón Chone.

\section{Materiales y métodos}

En lo que se refiere a la modalidad de la investigación concretamente se utilizó el paradigma denominado cuali-cuantitativo, donde la modalidad cualitativa permitió averiguar las cualidades o características generales del problema. Las características cualitativas de la problemática posteriormente son ratificadas mediante la cuantificación de la investigación de campo llevada a cabo generalmente en base a encuestas. Asimismo se utilizaron los métodos analítico - sintético que se aplicó en la revisión de la literatura y el inductivo - deductivo puesto que se indujo una solución particular al problema que luego puede ser deducida como una solución general.

Para este estudio se consideró una muestra conformada por un gerente, 15 clientes internos o personal de la entidad y 343 clientes externos o pacientes que utilizan los servicios de la fundación.

Sistema Informático para la gestión de servicios médicos. 
Rev. SINAPSIS, Vol. 7, Nº 1, Diciembre 2015

Una vez realizadas las encuestas se determinó la necesidad de desarrollar un sistema informático para la gestión de servicios médicos, para lo cual se utilizó la metodología Ciclo de vida o cascada, la cual quiere decir que en cada etapa se obtengan documentos que son las bases de partida de cada etapa siguiente, por lo tanto no puede comenzar antes de que se haya terminado la anterior.

\section{Resultados}

Una vez aplicadas las encuestas respectivas a los clientes internos de la entidad, se obtuvieron los siguientes resultados:

En lo referente a las recaudaciones económicas que se realizan por los diferentes servicios que presta la fundación.

\begin{tabular}{|l|r|r|}
\hline Respuestas & Frecuencia & Porcentajes \\
\hline Mediante Factura & 0 & 0.00 \\
\hline Mediante un registro electrónico & 0 & 0.00 \\
\hline Mediante recibos & 15 & 100.00 \\
\hline \multicolumn{1}{r|}{ TOTAL } & 15 & 100.00 \\
\hline
\end{tabular}

Tabla $\mathrm{N}^{\circ} 1$. Forma en la que se recaudan valores por concepto de servicios médicos.

Como se puede apreciar en la tabla $\mathrm{N}^{\circ} 1$ el $100 \%$ de los clientes internos afirman que actualmente las recaudaciones por los diferentes servicios que presta la fundación se lo realiza mediante recibos, lo que denota que dicho proceso debe ser realizado manualmente por la persona encargada de las recaudaciones

En lo que respecta a los medios utilizados para registrar las historias clínicas de los pacientes que se atienden en la fundación.

\begin{tabular}{|c|c|c|}
\hline Respuestas & Frecuencia & Porcentajes \\
\hline Física & 15 & 100.00 \\
\hline Digital & 0 & 0.00 \\
\hline Digital y física & 0 & 0.00 \\
\hline TOTAL & 15 & 100.00 \\
\hline
\end{tabular}

Tabla $N^{\circ}$ 2. Medios utilizados para registrar la información de historias clínicas

Sistema Informático para la gestión de servicios médicos. 
Rev. SINAPSIS, Vol. 7, No 1, Diciembre 2015

Como se puede apreciar en la tabla $\mathrm{N}^{\circ} 2$ el 100\% de los clientes internos afirman que la información de historias clínicas actualmente se maneja de forma física en fichas que una vez llenas son archivadas en una carpeta que se designa al paciente con su respectivo código de historia clínica, lo que denota una pérdida de tiempo en el momento de ingresar a un paciente ya que para verificar si este ya está ingresado tiene que acudir a un archivador y buscarlo por sus nombres lo cual es muy tedioso al momento en que la persona encargada de estadísticas tiene otros pacientes por ingresar.

Igualmente se aplicaron encuestas a los clientes externos, quienes respondieron de la siguiente manera:

En lo referente a la calificación que le otorgan al sistema actual de recaudación por concepto de servicios médicos.

\begin{tabular}{|c|c|c|}
\hline Respuestas & Frecuencia & Porcentajes \\
\hline Bueno & 30 & 8.75 \\
\hline Regular & 75 & 21.87 \\
\hline Malo & 238 & 69.39 \\
\hline TOTAL & 343 & 100.00 \\
\hline
\end{tabular}

Tabla $\mathrm{N}^{\circ}$ 3. Calificación al sistema actual de recaudación por concepto de servicios médicos

Como se puede apreciar en la tabla $\mathrm{N}^{\circ} 3$ el $8.75 \%$ de los encuestados manifiestan que el actual sistema de recaudación por los diferentes servicios ofertados es bueno, el $21.87 \%$ manifiesta que es regular, mientras que un $69.39 \%$ de los clientes externos lo califican de malo, situación que denota una tendencia a que el sistema muchas veces no ofrece información confiable y que requiere ser revisada debido al servicio diario que se ofrece, promoviendo pérdida de tiempo para el personal del departamento y principalmente para los usuarios externos.

En lo referente a la rapidez del sistema manual al momento de acceder a la información de historias clínicas.

\begin{tabular}{|l|r|r|}
\hline Respuestas & Frecuencia & Porcentajes \\
\hline Muy rápido & 0 & 0.00 \\
\hline Rápido & 0 & 0.00 \\
\hline Regular & 53 & 15.45 \\
\hline
\end{tabular}

Sistema Informático para la gestión de servicios médicos. 
Rev. SINAPSIS, Vol. 7, Nº 1, Diciembre 2015

ISSN 1390 - 9770

\begin{tabular}{|c|c|c|}
\hline Lento & 84 & 24.49 \\
\hline Demasiado lento & 206 & 60.06 \\
\hline TOTAL & 343 & 100.00 \\
\hline
\end{tabular}

Tabla $N^{\circ}$ 4. Rapidez para recuperar la información con el sistema manual

Como se puede apreciar en la tabla $\mathrm{N}^{\circ} 4$ el $15.45 \%$ de los encuestados percibe como regular el actual sistema para el manejo de historias clínicas, mientras que un $24.49 \%$ califica como lento el proceso para acceder a un historial clínico y un $60.06 \%$ manifiesta que el sistema que actualmente se aplica en el proceso para acceder a información de historias clínicas es demasiado lento.

Asimismo se encuestó a los pacientes sobre si la implementación de un sistema informático mejoraría la gestión de servicios médicos en la entidad, para lo cual se obtuvieron los siguientes resultados:

\begin{tabular}{|c|c|c|}
\hline Respuestas & Frecuencia & Porcentajes \\
\hline Si & 289 & 84.26 \\
\hline No & 0 & 0.00 \\
\hline No sabe & 54 & 15.74 \\
\hline TOTAL & 343 & 100.00 \\
\hline
\end{tabular}

Tabla $\mathrm{N}^{\circ}$ 5. Mejora en la gestión de servicios médicos mediante un sistema informático

Como se puede apreciar en la tabla $\mathrm{N}^{\circ} 5$ el $84.26 \%$ de los clientes externos manifiestan que si están de acuerdo con la implementación de un sistema informático para la gestión de servicios médicos mientras que el $15.74 \%$ de los clientes encuestados manifiestan no saben, situación que demuestra la clara necesidad de implementar un sistema informático que permita gestionar la información para mejorar el servicio brindado a los clientes de la Fundación Padre Miguel Fitzgerald.

Una vez que se aplicaron las encuestas correspondientes surgió la necesidad de crear un sistema informático para gestionar de mejor manera los servicios médicos, para lo cual se siguió el siguiente esquema.

Sistema Informático para la gestión de servicios médicos. 
Rev. SINAPSIS, Vol. 7, N 1, Diciembre 2015

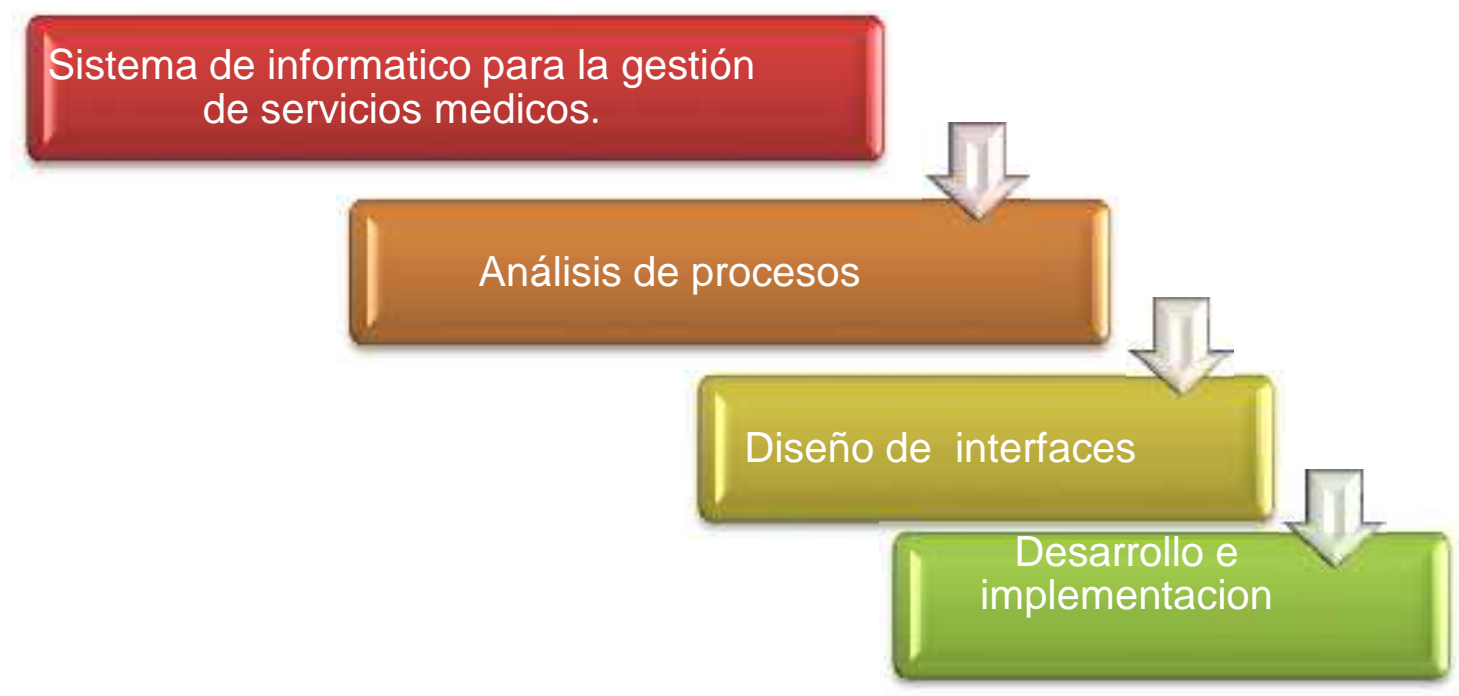

Figura $\mathrm{N}^{\circ}$ 1. Esquema re realización de la propuesta

En lo referente al análisis de procesos, una vez levantada la información y sugiriendo las mejoras pertinentes se obtuvieron los siguientes diagramas de procesos.

Compra de turnos

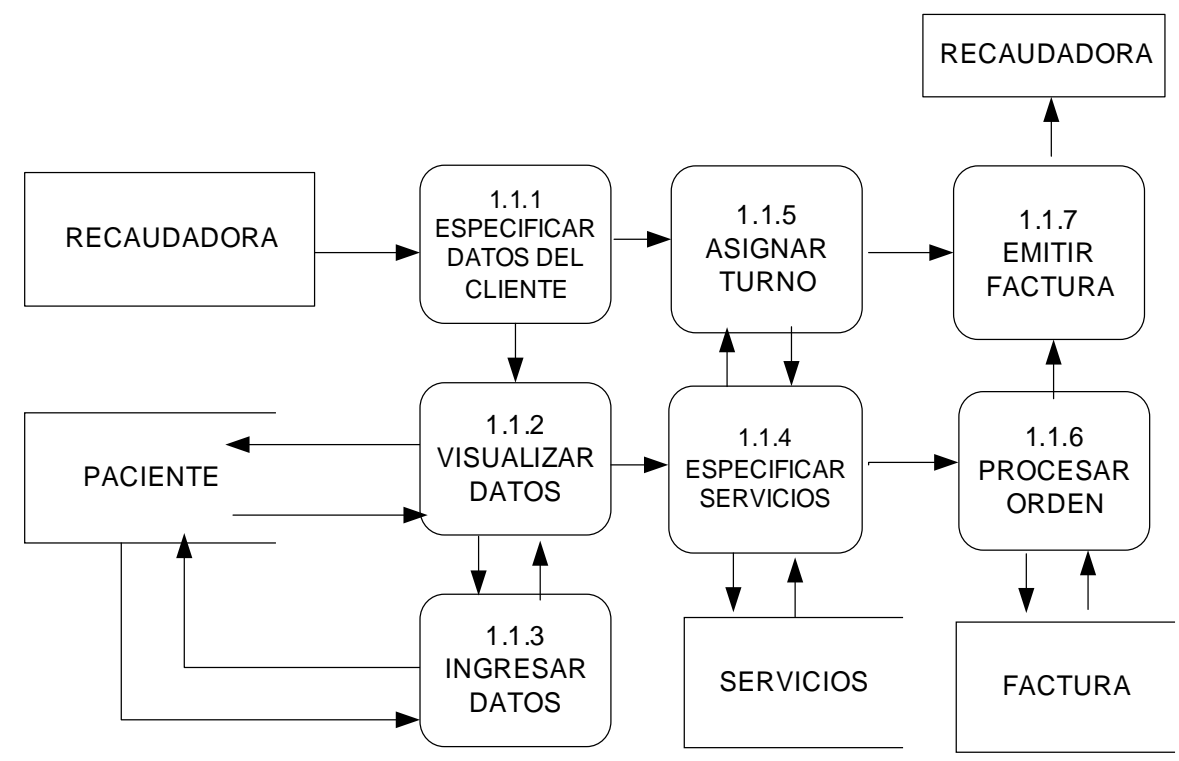

Sistema Informático para la gestión de servicios médicos. 
Rev. SINAPSIS, Vol. 7, N 1, Diciembre 2015

ISSN 1390 - 9770

Ingreso de datos

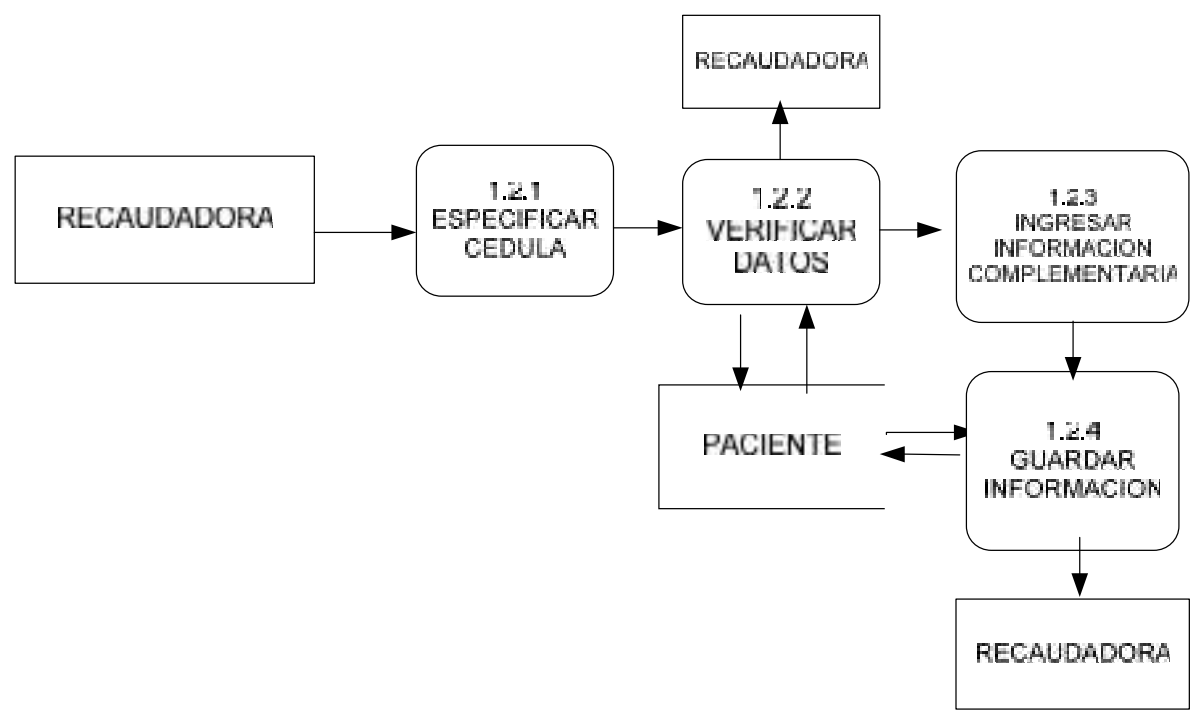

Emisión de reportes

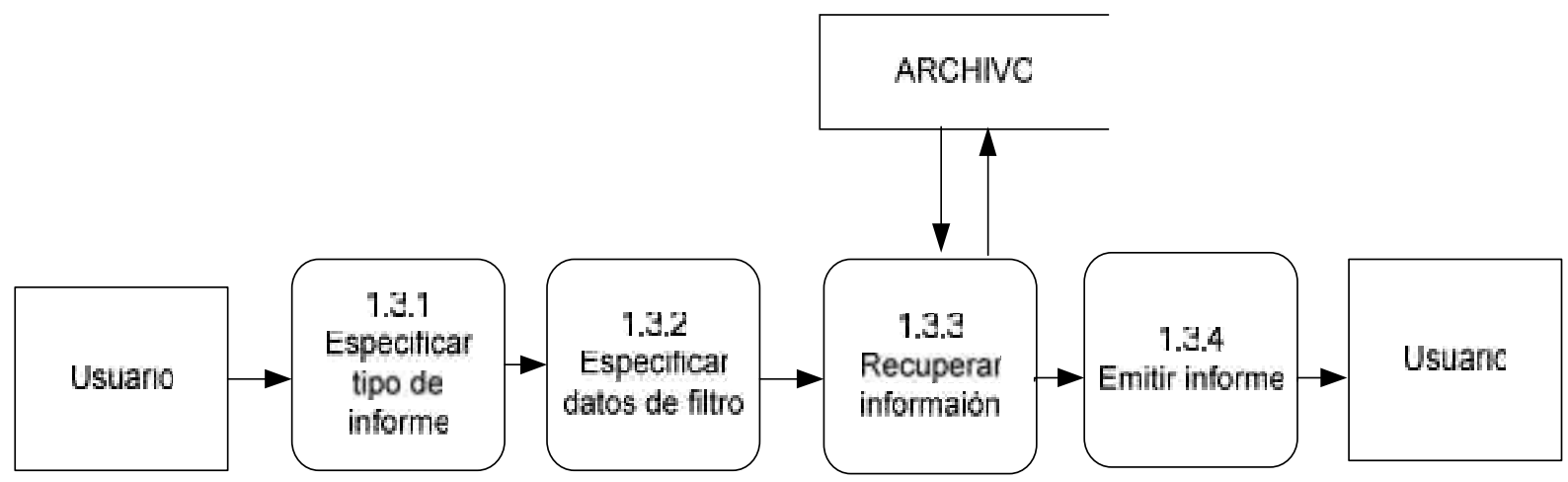

Asimismo se desarrollaron las siguientes interfaces:

Formulario de paciente.- Ingreso, actualización y consulta de datos de pacientes.

Sistema Informático para la gestión de servicios médicos. 
Rev. SINAPSIS, Vol. 7, Nº 1, Diciembre 2015

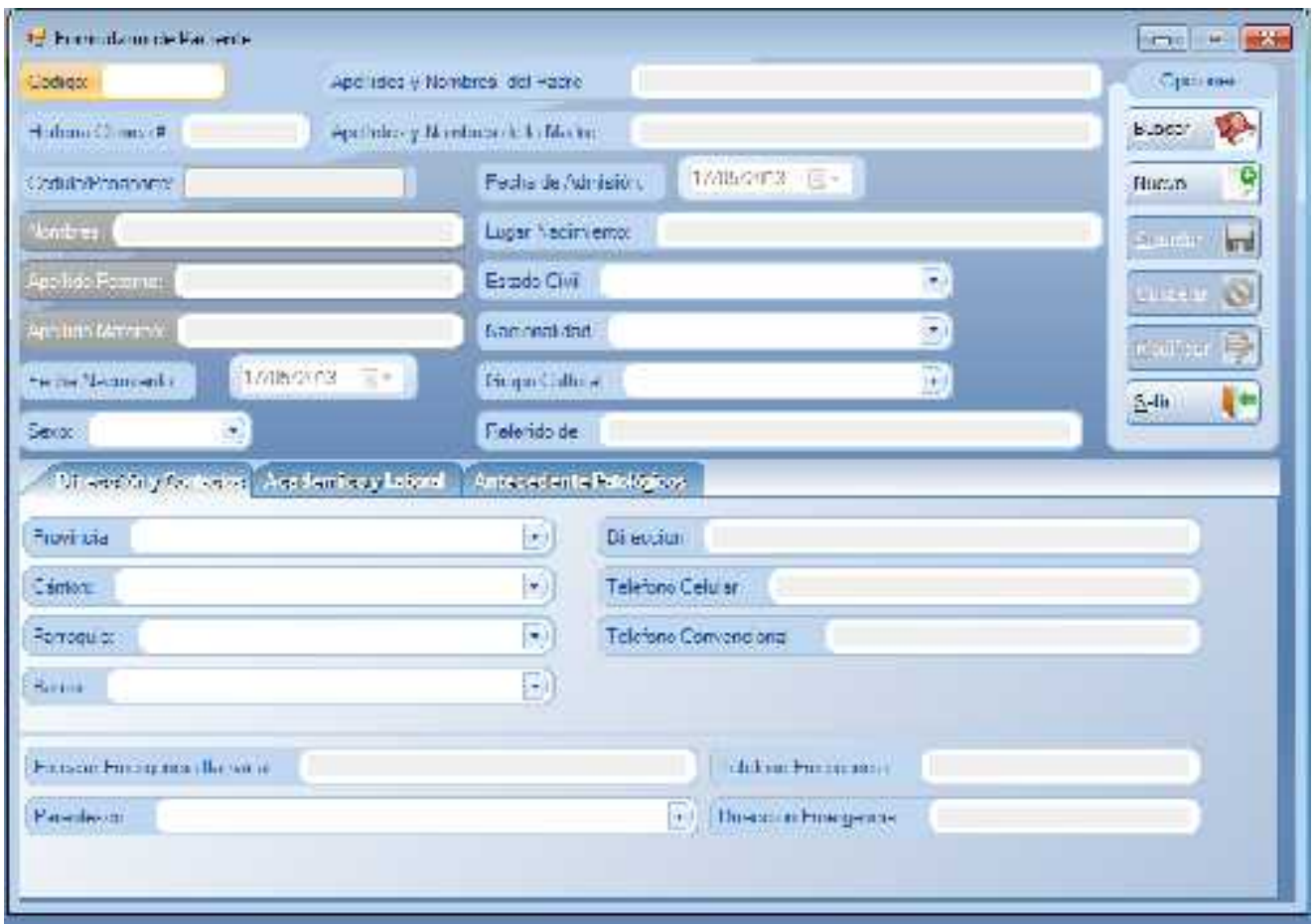

Figura $\mathrm{N}^{\circ}$ 2. Mantenimiento de datos de pacientes

Formulario de facturación.- Venta de servicios médicos.

Sistema Informático para la gestión de servicios médicos. 
Rev. SINAPSIS, Vol. 7, No 1, Diciembre 2015

ISSN 1390 - 9770

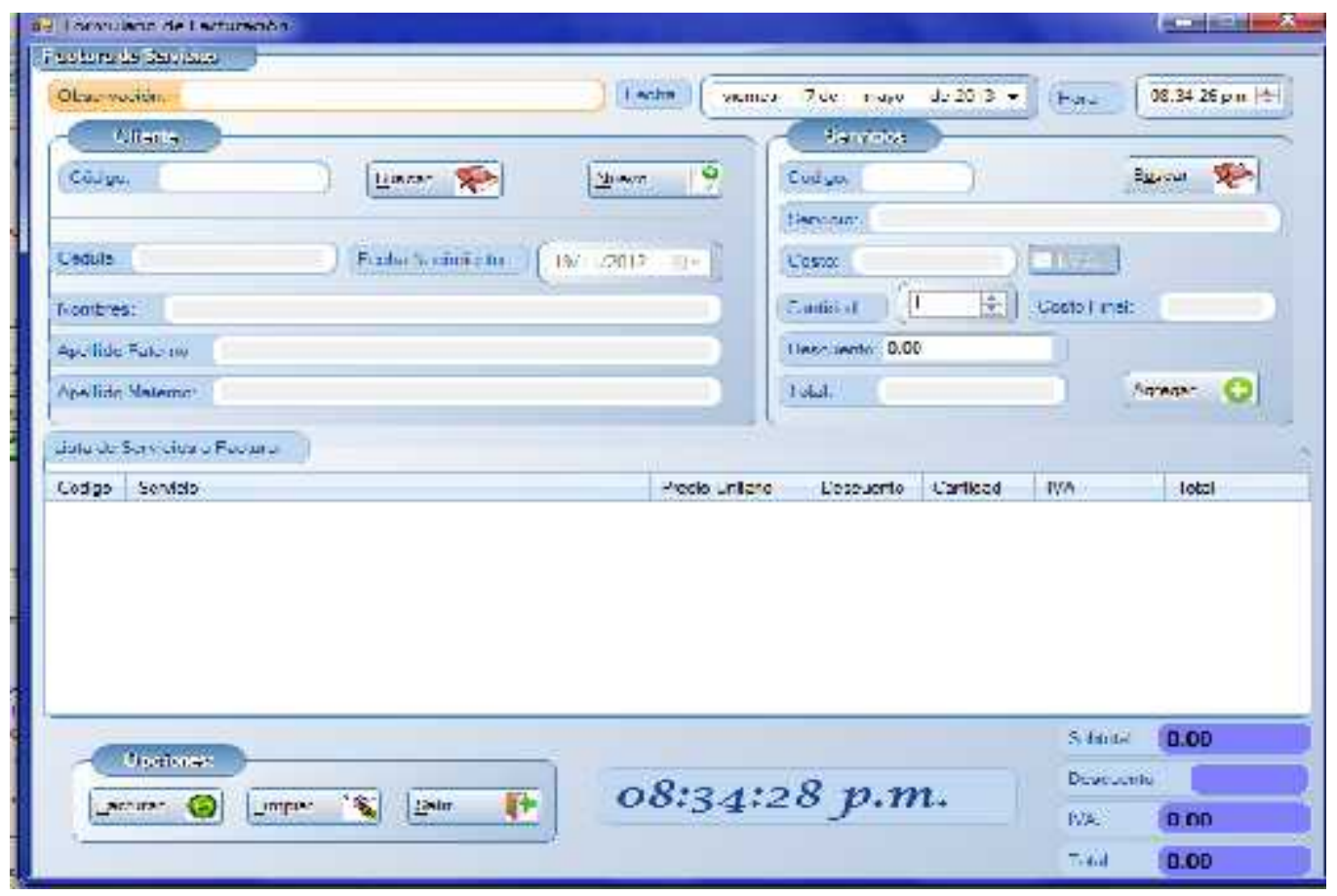

Figura $\mathrm{N}^{\circ} 3$. Venta de servicios

Estadísticas.- Emisión de estadísticas parametrizadas

Sistema Informático para la gestión de servicios médicos. 
Rev. SINAPSIS, Vol. 7, Nº 1, Diciembre 2015

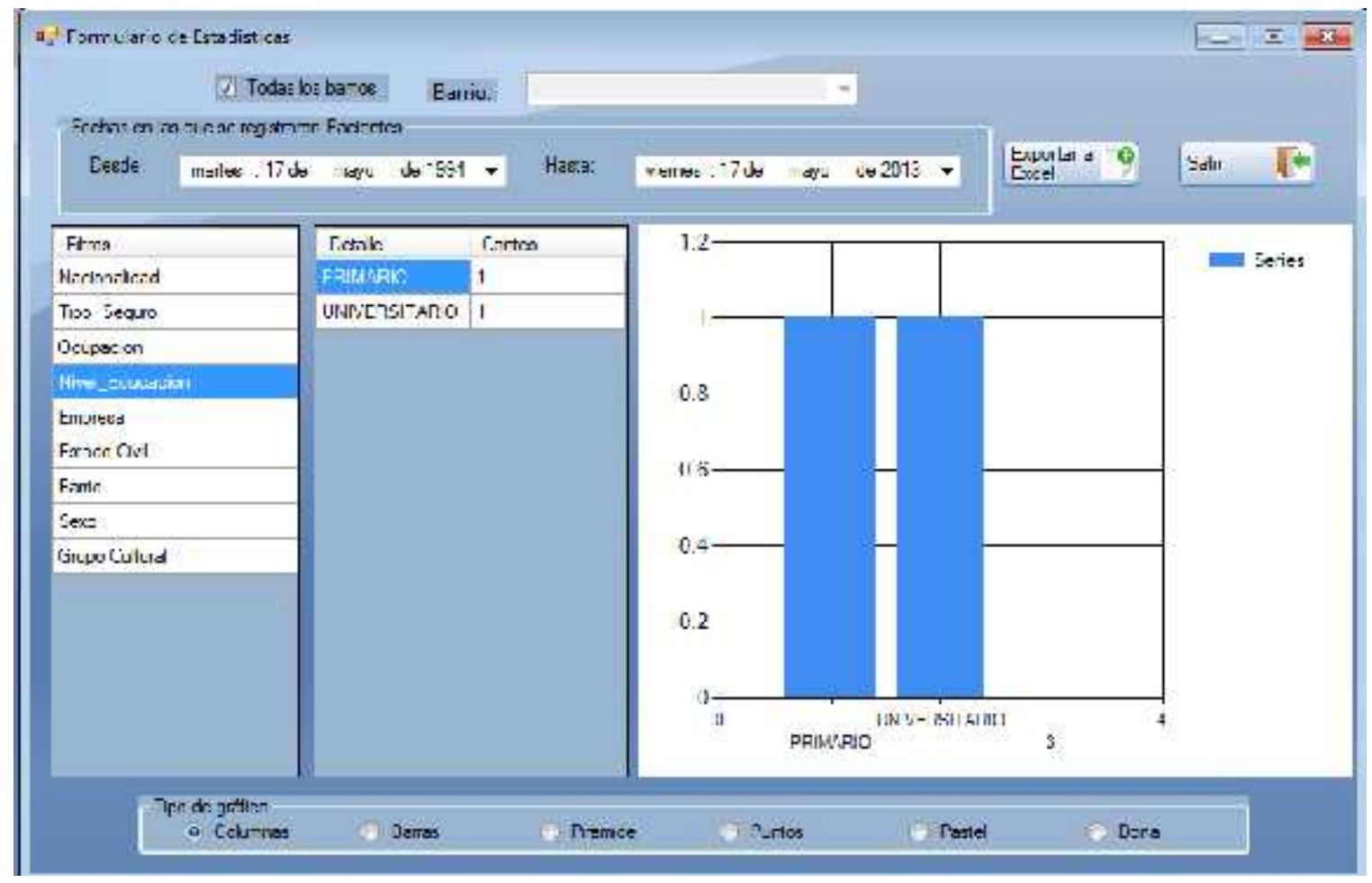

Figura $\mathrm{N}^{\circ}$ 4. Formulario de estadísticas

Una vez aplicado el sistema por un tiempo prudencial de seis meses, se realizó una nueva encuesta a los pacientes atendidos en base a la muestra inicial para conocer el nivel de satisfacción de los pacientes, obteniendo los siguientes resultados. 
Rev. SINAPSIS, Vol. 7, Nº 1, Diciembre 2015

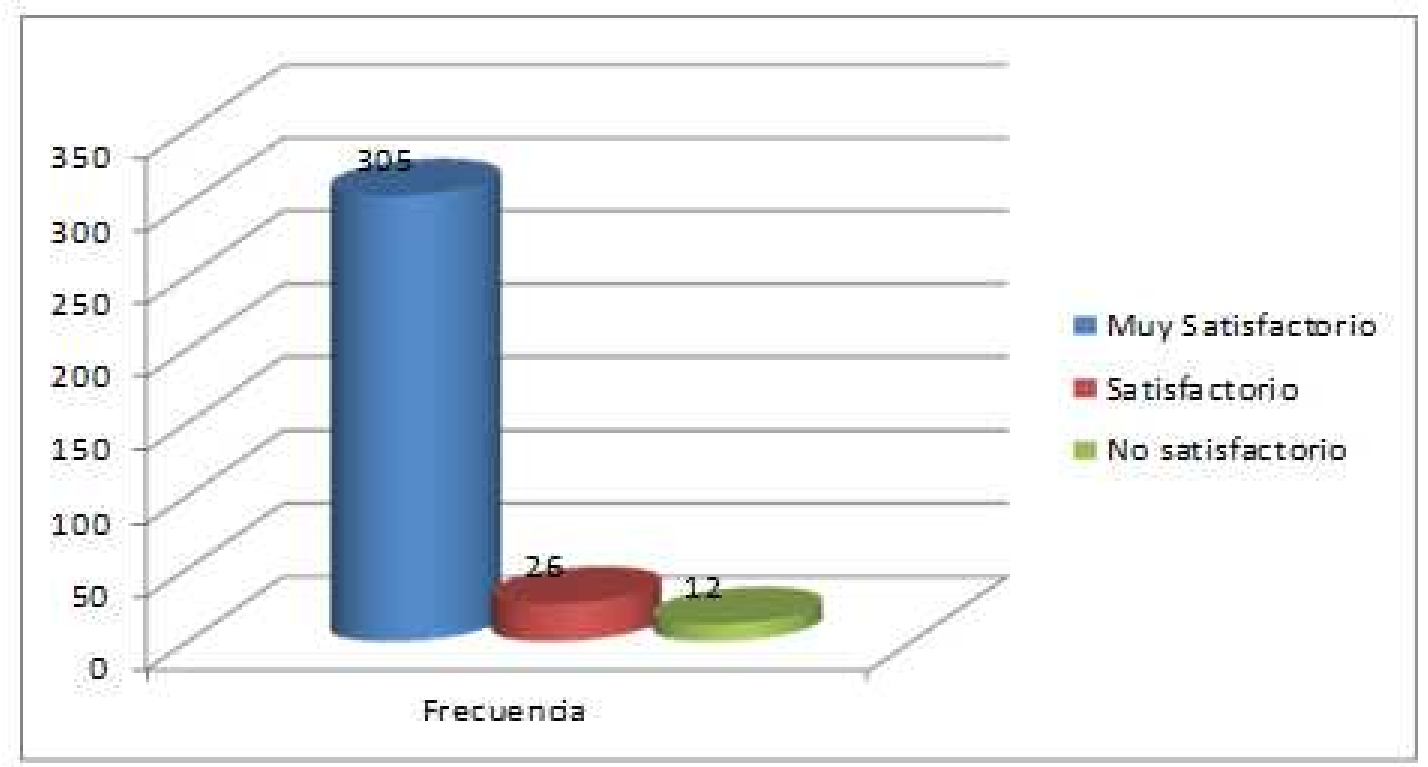

Figura $\mathrm{N}^{\circ}$ 5. Estadística de nivel de satisfacción de usuarios con el sistema implementado

Como se puede apreciar en la figura $\mathrm{N}^{\circ} 5$ el $88.92 \%$ de los pacientes manifestaron que los resultados de la implementación del sistema fueron muy satisfactorios, un 7\% manifestaron que los resultados han sido satisfactorios y sólo un 3.50\% han manifestado que los resultados son no satisfactorios.

\section{Discusión}

Los resultados obtenidos a partir de la encuesta inicial aplicada a los clientes internos y externos han permitido establecer varias necesidades que giraban en torno a las recaudaciones por servicios médicos, control de historias clínicas, emisión de reportes, generación de estadísticas, mantenimiento de la integridad de los datos, entre otros aspectos importantes, mostrando serias falencias que se derivan de un sistema manual.

Según (Lorenzo, Obed, Bembibre, \& Sánchez, 2013) La automatización de los sistemas de información constituye un aspecto de vital importancia para el correcto desenvolvimiento de disímiles actividades, por lo que el software, como equipamiento o soporte lógico de una computadora, ha devenido una herramienta esencial. Este representa el conjunto de componentes necesarios que permite la realización de tareas específicas. De ahí, la significación de los procesos vinculados a su desarrollo, los cuales se 
ocupan de todas las labores técnicas y de las gestiones básicas para crear y realizar un determinado procedimiento de forma eficaz.

A partir de las falencias encontradas inicialmente en esta investigación y debido a la intención de los involucrados para que se automaticen los procesos, se desarrolló un sistema informático ajustado a la realidad puntual de la Fundación Padre Miguel Fitzgerald del Cantón Chone, con la finalidad de ponerlo en funcionamiento por el lapso de seis meses, cuanto se aplicó dicha estructura se puedo evidenciar el ahorro de tiempo en la captura de datos y en la recuperación de la información, asimismo se facilitó el registro de la información mediante validaciones más precisas que permitieron garantizar la integridad de la información registrada, con estos resultados también se permitió generar estadísticas precisas a partir de las historias clínicas, proveyendo de información importante para que los directivos de la entidad puedan tomar decisiones con un soporte informático confiable para dicha tarea.

Como lo afirma (Señor, 2000) La necesidad de archivar y analizar datos en las instituciones de salud tanto en el nivel primario como en el secundario y terciario es trascendental para evaluar las tendencias de salud en la población, por este motivo se ha incorporado un módulo de estadísticas en la aplicación desarrollada para tener datos al instante sobre las tendencias de los pacientes en este ámbito.

La herramienta desarrollada ha aportado con un mecanismo confiable, rápido y preciso para gestionar la información de los pacientes de la entidad en mención, evitando inconformidades a los usuarios tanto internos como externos. La particularidad de la aplicación es que integra los servicios médicos que presta la entidad con la gestión de cobros que realiza por concepto de dichos servicios prestados, esta afirmación concuerda con el criterio de (Marín, 2009) quien afirma que desde hace varias décadas la informática ha encontrado en la medicina una de sus aplicaciones más comunes e importantes que ha permitido al sector de la salud, no sólo contar con métodos novedosos, sencillos y eficaces de gestión administrativa en consultas, hospitales y centros de investigación biomédica, sino también disponer de recursos informáticos de gran valor en las exploraciones con tratamiento de imágenes, señales bioeléctricas y otras que reducen la posibilidad de error en el diagnóstico de las enfermedades. 
Para finalizar se llevó a cabo una encuesta de satisfacción a los usuarios, quienes expresaron casi en un 89\% que la implementación del sistema ha sido muy satisfactoria, promoviendo el ahorro de tiempo, la optimización de recursos y la integridad de la información que se maneja en el mismo. Estos resultados concuerdan con el criterio de (Curioso, Saldías, \& Zambrano, 2002) quienes manifiesta que el valor potencial de las computadoras en medicina ha sido reconocido por años y la informática médica ha emergido como un campo de investigación interdisciplinaria importante en medicina. Avances en la tecnología de las historias clínicas electrónicas (HCE) han hecho posible que las HCE reemplacen muchas funciones de la tradicional historia clínica en papel y el uso de HCE promete logros significativos en el cuidado del paciente.

\section{Conclusiones}

Una vez culminada esta investigación se afirma que la gestión de servicios médicos debe estar soportada por aplicaciones informáticas que de preferencia tienen que estar construidas a la medida de las necesidades del problema analizado, puesto que es allí donde se puede explotar de mejora manera los recursos tecnológicos y de esta manera se mantendrá en un nivel alto la satisfacción de los usuarios que se hagan atender en las entidades de salud. Es necesario destacar que las HC deben ser registradas en medios electrónicos, puesto que la información ya no es local, sino global, y los datos que se registran en ella deben estar a disposición de los usuarios en todo lugar y momento, puesto que esto puede marcar la diferencia entre la vida y la muerte de una persona en un momento determinado. Por otra parte para los directivos es fundamental contar con información clara, precisa, relevante y oportuna, ya que son ellos quienes deben tomar las decisiones más neurálgicas en torno a la administración de una unidad de salud, para esto deben contar con sistemas que brinden dicha información de acuerdo a los parámetros citados anteriormente.

\section{Bibliografía}

1. Avella, L., \& Parra, P. (2013). Tecnologías de la Información y la Comunicación (TICS) en el sector salud. Bogotá. 
Rev. SINAPSIS, Vol. 7, Nº 1, Diciembre 2015

ISSN 1390 - 9770

2. Cabañas, J., Scholer, M., \& Tintinalli, J. (2008). Informática en Medicina de urgencias y emergencias: Gestión de la Información y aplicaciones en el siglo XI.

3. Curioso, W., Saldías, J., \& Zambrano, R. (2002). Historias clínicas electrónicas. Experiencia en un hospital nacional. Satisfacción por parte del personal de salud y pacientes.

4. Llanusa, S., Rojo, N., Caraballoso, M., Capote, R., \& Perez, J. (2005). Las tecnologías de la información y comunicación y la gestión del conocimiento en el sector de la salud. La Habana.

5. Lorenzo, M., Obed, A., Bembibre, D., \& Sánchez, M. (2013). Diseño e implementación del sistema de información interna para el Departamento de Imaginología del Hospital Provincial "Manuel Ascunce Domenech", de Camagüey. La Habana.

6. Marín, M. (2009). Capacitación en el área de la informática en salud.

7. Ribelles, N., Jeréz, J., Urda, D., Subirats, J., Márquez, A., Quero, C., . . Franco, L. A. (2010). Galén: Sistema de Información para la gestión y coordinación de procesos en un servicio de Oncología. Málaga.

8. Señor, R. (2000). La informática médica en Cuba.

9. Stusser, R., \& Rodríguez, A. (2006). La informatización de la atención primaria de salud. La Habana.

Sistema Informático para la gestión de servicios médicos. 